\title{
Relaciones Proceso, Microestructura, Propiedades del Cromado Duro a Escala de Laboratorio
}

\author{
Juan D. Ordóñez Barriga ${ }^{1 *}$, Alfredo Valarezo ${ }^{1}$ \\ ${ }^{1}$ Universidad San Francisco de Quito - Colegio de Ciencias e Ingenierías. Diego de Robles y Via Interoceánica, Cumbayá-Ecuador. \\ *Autor principal/Corresponding author, e-mail: juan_ord@live.com \\ Editado por/Edited by: Cesar Zambrano, Ph.D. \\ Recibido/Received: 2015/04/19. Aceptado/Accepted: 2015/09/27. \\ Publicado en línea/Published on Web: 2015/12/30. Impreso/Printed: 2015/12/30.
}

Relationships Process, Microstructure, Properties of Hard Chromium Plating in Laboratory Scale

\begin{abstract}
In this work hard chromium electroplating process is developed on small test specimens on AISI A36 steel. This paper includes the development of hard chrome plating process at laboratory scale and provide a simplified procedure for industrial applications. By applying a differential power over the A36 steel submerged in an electrolytic bath of chromic acid, a thin coat of 30 to 80 microns of chrome is deposited over the specimen. The hard chrome deposition is achieved by a combination of multiple variables such as: chromic acid concentration, substrate metal, anodes, current density, solution temperature and time. From the abovementioned parameters, the time and the current density are the working parameters to be manipulated while the others remain unchanged. For these experiment, a linear simplified model is theoretically and experimentally derived from Faraday's electro-deposition law for hard chrome plating. The thickness variation, micro-hardness and cracking network of the combination of the working parameters are included and discussed in this work. The chrome deposit obtained is microscopically and gravimetrically measured. The results obtained by combining the working parameters show hardness values within the range provided of 66 to $70 \mathrm{HRC}$. This paper studies and analyzes the possible causes that influence hardness with the microstructure (number of surface cracks, density) and processing parameters such as time and current density.
\end{abstract}

Keywords. Hard Chrome Plating, Faraday law, microhardness, microcracks, time, current density.

\section{Resumen}

En el presente trabajo se desarrolla experimentalmente el proceso de cromado duro sobre probetas de acero AISI A36. El trabajo incluye el desarrollo del proceso de cromo duro a nivel de laboratorio para brindar un procedimiento simplificado en la aplicación industrial. La electro-deposición es el principio fundamental de depósito del cromo duro, mediante la aplicación de un diferencial de potencial eléctrico, sobre el acero A36 sumergido en un baño electrolítico de ácido crómico, se deposita una capa de cromo entre 30 - 80 micrómetros. El depósito de cromo se logra mediante la combinación de variables; tales como: concentración de la solución electrolítica de ácido crómico, material base, ánodos, densidad de corriente, temperatura de la solución y tiempo. De las anteriores, el tiempo y la densidad de corriente son los parámetros de trabajo que serán manipulados mientras los otros permanecerán constantes. Un modelo simplificado lineal derivado de la ley de electro-deposición de Faraday es desarrollado. Se presenta la variación del espesor de material depositado, micro-dureza y agrietamiento del depósito de cromo duro según la combinación de parámetros de tiempo y densidad de corriente empleados. El espesor del $\mathrm{Cr}$ depositado es medido gravimétricamente y microscópicamente. Los resultados obtenidos al combinar dichos parámetros muestran una estabilización de valores de dureza dentro del rango de 66 a 70 HRC. Esta investigación estudia y analiza las posibles causas que influencian la dureza relacionada al número de grietas superficiales, tiempo y densidad de corriente en el proceso de cromado duro.

Palabras Clave. Recubrimiento de Cromo Duro, ley de Faraday, microdureza, microgrietas, tiempo, densidad de corriente. 


\section{Introducción}

Durante las últimas décadas, los recubrimientos de cromo duro han sido empleados en grandes industrias a nivel mundial gracias a sus destacadas propiedades. Sus aplicaciones son diversas debido a los beneficios que este ofrece. Su principal ventaja es alta dureza superficial, variable entre 65 - 70 en escala Rockwell C [1]. La dureza es esencial para prolongar el tiempo de vida de las partes mecánicas móviles que se encuentran sometidas en un ambiente con constantes tensiones y altos esfuerzos. Por ello el recubrimiento es empleado por ejemplo en: anillos de pistón de motor, amortiguadores, ejes, barras hidráulicas, cilindros entre otros.

El recubrimiento de cromo duro consiste en el depósito electro químico de una capa de cromo sobre un material substrato con propiedades ferromagnéticas. Las características de dicha capa depositada posee propiedades de alta dureza, anti-corrosiva y una apariencia brillante agradable a la vista. Las aplicaciones del cromado duro son extensas, según la Sociedad Americana de Metales (ASM por sus siglas en inglés) el cromo duro busca aumentar el tiempo de servicio de partes funcionales agregando un recubrimiento superficial con bajo coeficiente de fricción resistente al desgaste y adicionalmente, busca restaurar dimensiones de partes que fueron reducidas en tamaño por el deterioro con alta dureza como complemento [2]. Resistencia a la corrosión es la segunda característica importante que brinda el recubrimiento superficial de cromo duro. La resistencia a la corrosión es obtenida a partir el depósito ordenado de conjuntos de moléculas de cromo alineados de forma cristalina y que a su vez es depositada uniformemente. Sin embargo, estos conjuntos de moléculas son delimitados por grietas creadas por átomos de hidrógeno generados durante la electrolisis; se filtran en el material entre los enlaces químicos del depósito y conllevan a la creación de puentes falsos entre la unión de átomos de cromo, puntos de baja resistencia, y concentradores de esfuerzo propensos a fractura [3].

El éxito alcanzado en el proceso de electro-deposición de cromo duro sobre el material substrato se dio gracias a la manipulación controlada de dos variables principales: corriente y tiempo. Se trabajó con una fuente de poder de alta precisión con una ondulación de voltaje continuo bajo el $5 \%$ al trabajar con un amperaje fijo. La aplicación de energía eléctrica sobre el área de la pieza de ensayo produjo una densidad de corriente que se rige por la ley de Faraday y correlaciona la carga suministrada con la cantidad de masa depositada; mientras que el espesor del depósito varió de acuerdo al tiempo de encendido de la fuente conectada con la pieza dentro del baño electrolítico.

Dada la importancia del rol que juegan las variables de corriente y tiempo, el objetivo principal de esta investigación es desarrollar las relaciones proceso, microestructura, propiedades del cromado duro a escala de laboratorio para obtener una mejor estabilidad y control sobre el proceso. Se tiene como meta evaluar los efectos de la corriente y tiempo de exposición en el proceso de cromado, caracterizar la microestructura basada en los posibles efectos del procesamiento mediante análisis del espesor, inspección metalográfica, densidad de grietas y evaluar la correlación teórica-experimental de los resultados de carga eléctrica, espesor del depósito y la eficiencia del cromado duro.

\section{Métodos}

La energía eléctrica, base requerida para efectuar el proceso de cromo duro, fue utilizada primordialmente para la separación de los enlaces químicos de moléculas que componen el ácido crómico, obtener cromo y lograr el depósito sobre acero A36. La aplicación de energía dentro del baño electrolítico generó reacciones electroquímicas y calor. El cromo hexavalente, presente en el ácido crómico, separado del hidrogeno y del oxígeno debe ser reducido y adherido a la superficie a recubrir. Para lograr la reducción se requiere la presencia de gran cantidad de electrones, romper su previo enlace, reducir y adherir el cromo sobre la pieza a recubrir. Con el fin de explicar de mejor manera lo anteriormente mencionado se resuelve las ecuaciones de reducción.

La fórmula molecular en estado estable del ácido crómico está dada por:

$$
2 \mathrm{H}_{2} \mathrm{CrO}_{4} \rightarrow \mathrm{H}_{2} \mathrm{Cr}_{2} \mathrm{O}_{7}+\mathrm{H}_{2} \mathrm{O}
$$

El primer término se refiere al ácido crómico en su estado estable mientras que el segundo y tercer término representa el mismo ácido expandido para una mejor explicación. Si se observa el segundo término y si a este se le introducen doce (12) electrones se logra romper el enlace de formulación estable y obtener cromo metal $\left(\mathrm{Cr}^{0}\right)$ a partir de la reducción del cromo hexavalente $\left(\mathrm{Cr}^{6+}\right)$. Cabe recalcar que esta reacción sucede sobre la superficie del substrato que es cargada negativamente y el cromo es adherido a la misma. Se desarrolla lo anteriormente mencionado para una mejor visualización de lo ocurrido:

$$
\left(\mathrm{Cr}_{2} \mathrm{O}_{7}\right)^{-2}+12 e^{-}+14 \mathrm{H}^{+} \rightarrow 2 \mathrm{Cr}^{0}+7 \mathrm{H}_{2} \mathrm{O}
$$

Sin embargo, no siempre se logra reducir instantáneamente el cromo hexavalente con doce electrones y causar su adhesión sobre la superficie del substrato, sino que se reduce únicamente con tres (3) electrones y se presenta la formación del ion cromo trivalente $\left(\mathrm{Cr}^{3+}\right)$; sólido que precipita que no se adhiere al material. A pesar de ello, si el cromo trivalente logra alcanzar el nodo positivo, transfiere nuevamente los electrones y conduce a la formación de cromo hexavalente tal como se muestra a continuación:

$$
\left(\mathrm{Cr}_{2} \mathrm{O}_{7}\right)^{-2}+6 e^{-}+14 \mathrm{H}^{+} \rightarrow 2 \mathrm{Cr}^{+3}+7 \mathrm{H}_{2} \mathrm{O}
$$




$$
C r^{3+}-3 e^{-} \rightarrow C r^{+6}
$$

Ambos iones: cromo trivalente y hexavalente $\left(\mathrm{Cr}^{3+}\right.$, $\left.\mathrm{Cr}^{6+}\right)$ son sumamente tóxicos y cancerígenos para la salud humana [4]. Por lo que siempre se sugiere una adecuada manipulación y buen manejo de las emisiones durante el procesamiento del cromado.

Es fundamental resaltar que al reducir el ácido crómico con doce (12) electrones, del segundo término de la ecuación (1), se desprenden dos iones de hidrogeno que en combinación a dos electrones genera gas hidrogeno. La reacción mencionada se resuelve a continuación:

$$
2 H^{+}+2 e^{-} \rightarrow H_{2} \text { (Gas hidrogeno generado) }
$$

La formación de átomos de hidrógeno durante la electrodeposición resulta peligroso debido a que dichos átomos se posicionan entre los enlaces químicos causando fragilización por hidrógeno. Esto hace que la unión de los átomos se debilite causando puntos de baja resistencia y concentradores de esfuerzo propensos a fractura [3]. Para prevenir que exista falla debido a fragilización por hidrógeno se recomienda primero, prevenir con inhibidores químicos la absorción de hidrógeno, segundo, se puede eliminar esfuerzos residuales antes de la electrodeposición, y tercero, se recomienda remover átomos de hidrógeno mediante tratamiento térmico luego del proceso de electro-deposición [5].

\section{Materiales y equipos}

La selección del material base, la solución electrolítica de ácido crómico, ánodos y temperatura de la solución fueron constantes, mientras que la densidad de corriente y tiempo fueron variados controladamente. Los equipos de trabajo de laboratorio fueron: calentador y agitador magnético, fuente de potencial eléctrico, cables eléctricos y equipo de medición de dureza.

El material substrato fue elaborado a partir de acero A36, probetas rectangulares de $35 \times 20 \times 3[\mathrm{~mm}]$. Su dureza teórica es de 149 Brinell Hardness equivalente a 80 Rockwell B [6]. Se perforó un agujero en la parte superior de la probeta para sujeción.

La solución de ácido crómico es categorizada como baño de catalizadores mixtos, compuesta por ácido crómico y tres aditivos catalizadores especiales: CRA, CRB y CRC [7].

Se utilizó un calentador automático y agitador magnético de laboratorio marca Nuova para mantener la temperatura uniforme de la solución. Además, se empleó una fuente de poder marca Agilent para suministrar energía eléctrica. El diámetro de los cables se determinó a partir de la tabla de dimensionamiento de cables de American Wire Gauge (AWG por sus siglas en inglés) con un área transversal de $2.61\left[\mathrm{~mm}^{2}\right]$ para el amperaje de siete (7) amperios.

Los micro-durómetros: marca Wilson Tukon y Buehler fueron empleados para realizar el análisis de microdureza superficial y transversal respectivamente. El microdurómetro Wilson Tukon trabajó con punta de diamante tipo Knoop y $500 \mathrm{~g}$ de carga. Mientras que el micro durómetro Buehler trabajó con punta de diamante piramidal Vickers y 300g de carga. El cálculo de microdureza Knoop fue realizado mediante la determinación de la longitud mayor de la indentación de la punta de diamante sobre el metal; mientras que el cálculo de micro dureza Vickers fue realizado mediante la suma de ambas diagonales y obtener el promedio de la incisión piramidal realizado sobre el recubrimiento de cromo. La fórmula para la obtención de la micro dureza para ambos casos se obtuvo a partir de la normativa ASTM E 384 "STANDARD TEST METHOD FOR MICROINDENTATION HARDNESS OF MATERIALS”, aplicando el factor de corrección ocular propia del equipo [8].

\section{Desarrollo}

Las probetas fueron preparadas y pulidas mecanográficamente con lijas de granulación 80, 180, 600, 1000, 1200 y 1500 por pulgada cuadrada según la numeración estándar de la American National Standard Institute (ANSI por sus siglas en ingles). Posteriormente, las probetas fueron desengrasadas y limpiadas superficialmente con la solución desengrasante comercial CT-10.

\section{Depósito de Cromo}

El armado de conexiones para el proceso de cromado inició con la conexión de la probeta hacia la fuente eléctrica de manera catódica debido a que fue requerido electrones (carga negativa) sobre la superficie de la probeta. La temperatura de la solución se mantuvo constante a cincuenta y cinco grados centígrados $\left(55^{\circ} \mathrm{C}\right)$ y se manipuló controladamente el tiempo (30, 60, $90 \mathrm{mi}-$ nutos) junto con la densidad de corriente $(39,48$ y 57

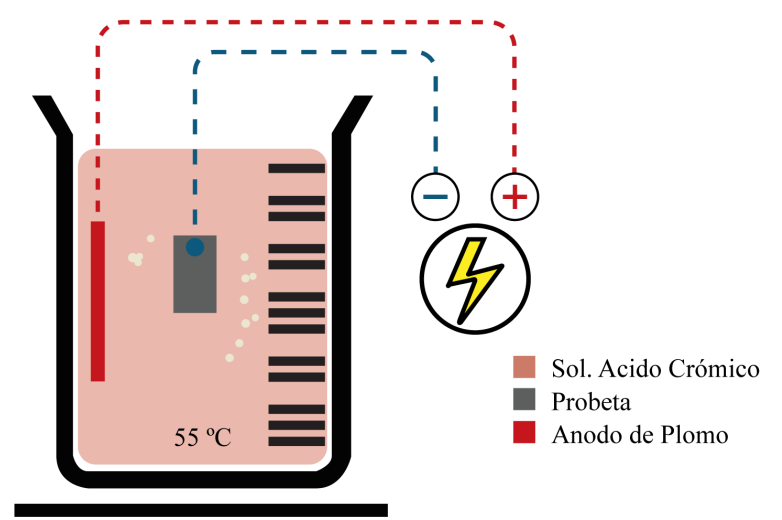

Figura 1: Conexiones y armado de equipo para baño electrolítico de Acido Crómico. 


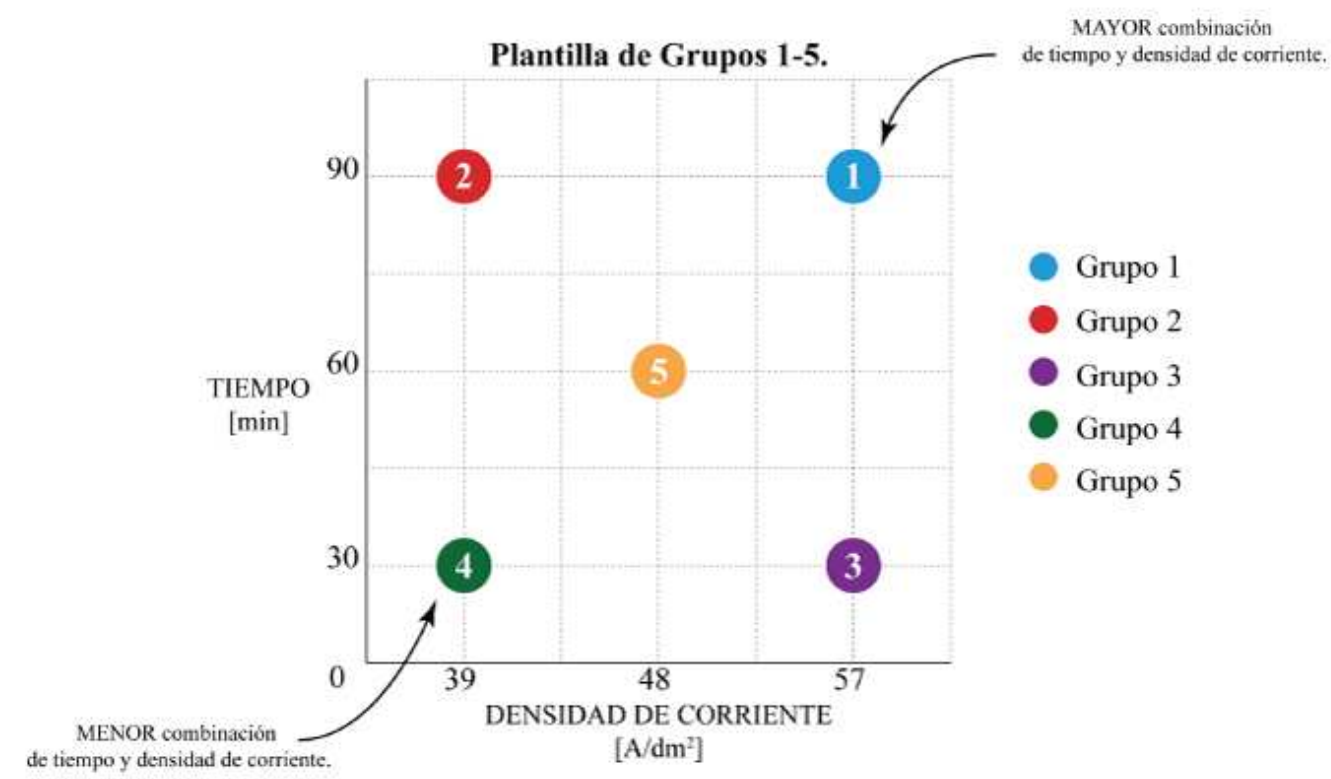

Figura 2: Diseño de experimentos, plantilla de condición central y condiciones-frontera. Combinaciones de tiempo y densidad de corriente acopladas en grupos 1-5.

$\mathrm{A} / \mathrm{dm}^{2}$ ). La conexión realizada se muestra en la Figura 1.

Para una visión clara de los resultados al manipular los parámetros de tiempo y densidad de corriente se diseñó una matriz que limitó las condiciones de ensayo. Cinco (5) combinaciones de parámetros fueron creados a los cuales se los denominó grupos. Cada grupo fue conformado por tres (3) probetas ensayadas con los mismos parámetros. La matriz de combinación de parámetros se muestra en la Figura 2 y los parámetros utilizados para cada grupo se muestra en la Tabla 1.

\begin{tabular}{|c|c|c|}
\hline Grupo $\mathbf{N}^{\circ}$. & $\begin{array}{c}\text { Densidad de } \\
\text { Corriente }\left[\mathrm{Amp} / \mathrm{dm}^{2}\right]\end{array}$ & Tiempo [min] \\
\hline 1 & 57 & 90 \\
\hline 2 & 39 & 90 \\
\hline 3 & 57 & 30 \\
\hline 4 & 39 & 30 \\
\hline 5 & 48 & 60 \\
\hline
\end{tabular}

Tabla 1: Parámetros de caracterización, tiempo y densidad de corriente, para los grupos 1-5.

\section{Análisis metalográfico y de micro-dureza}

Para hacer por un lado las mediciones de dureza eficientes y aprovechar por otro lado la determinación de grietas creadas según los diferentes parámetros, se requiere análisis destructivo y ataque químico de las probetas. Entonces, para el análisis metalográfico fue plasmado el mismo procedimiento de ensayo destructivo para cada grupo de probetas. El procedimiento de corte, desbaste y pulido probetas se realizó de acuerdo a la normativa ASTM E3-01 "GUIDE FOR PREPARATION OF METALLOGRAPHIC SPECIMENS" [9]. Se efectuó un corte transversal a tres (3) milímetros sobre la parte inferior de la probeta. Luego se utilizó un banco de pulido grueso para desbaste de probetas con lijas de granulación ANSI: 600, 1000, 1200 y 1500. La probeta fue lijada según la Tabla 5 de la normativa ASTM E301 durante 45 segundos por cada numeración de tamaño de grano ANSI. Posteriormente se empleó el banco de pulido fino, con paño medio y pasta de diamante según especificado dentro de dicha norma.

Para la determinación de grietas y una mejor observación bajo el microscopio, las probetas ensayadas fueron atacadas químicamente con una solución de ácido nítrico con $95 \%$ de pureza, y ácido clorhídrico con $37 \%$ de pureza. El ataque químico se realizó durante 5 segundos bajo los parámetros de trabajo detallados en la norma ASTM E407 "PRACTICE FOR MICROETCHING METALS AND ALLOYS" [10].

\section{Resultados}

A partir de los parámetros de tiempo y densidad de corriente combinados se presentan los resultados de la inspección visual, medición gravimétrica del depósito de cromo, comportamiento del depósito según la ley de Faraday junto con la eficiencia del proceso, análisis metalográfico que comprende de análisis de espesor, microdureza y agrietamiento del cromo depositado.

\section{Inspección Visual}

El acabado superficial intervino significativamente en el depósito de cromo sobre las probetas de acero A36. Para demostrar este hecho la Figura 3 muestra las probetas No. 12 y No. 19. Lo particular de éstas es que se utilizó diferente grado de desbaste para el acabado superficial pero se empleó los mismos parámetros en ambas. La Figura 3 tiene una ampliación de 10x y degrada el color base para resaltar depósitos nodulares creados debido a la superficie rugosa. 


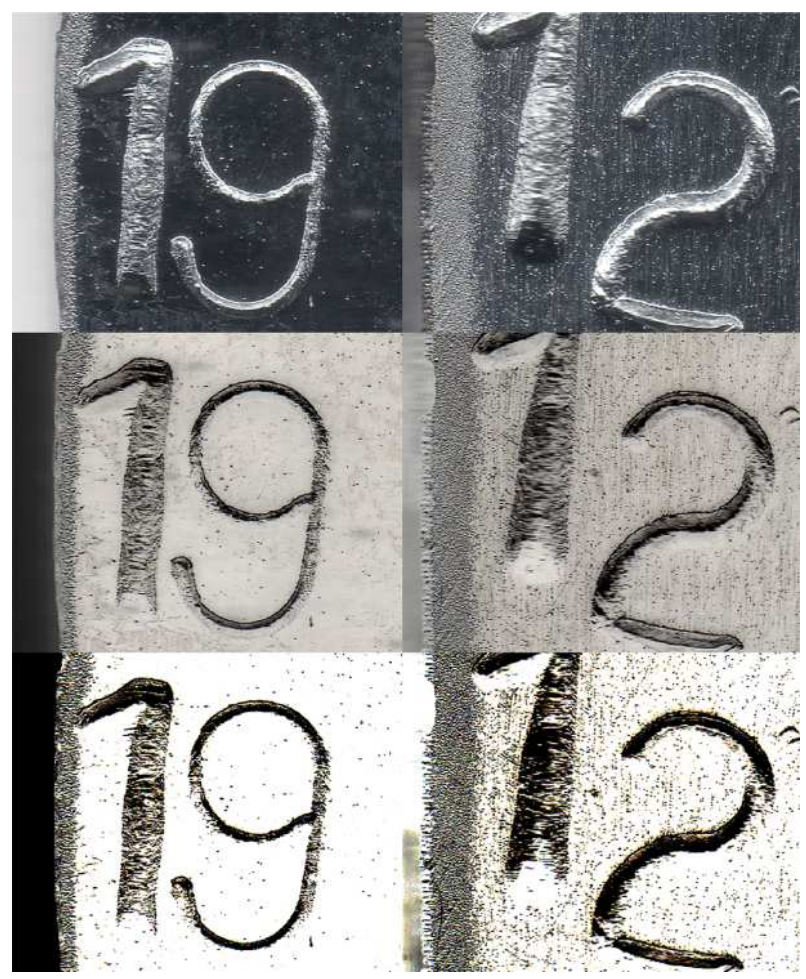

Figura 3: Depósito nodular sobre las probetas \#12 y \#19. Se aplica degradado gráfico de color base (hacia abajo) para una mejor observación de la cantidad de depósitos nodulares sobre la superficie.

Se observa en la parte inferior de la Figura 3 que el degrade de color actúa completamente, el depósito nodular sobre la superficie de la probeta No. 12 es de fácil visualización y resultó 6.7 veces mayor (medido por análisis de imagen) que el depósito nodular de la probeta No. 19. Las esquinas y picos creados por la rugosidad del acabado superficial actuaron como concentradores de electrones que facilitaron la separación del enlace de cromo y se depositó el material más frecuentemente sobre estos puntos [2].

\section{Medición gravimétrica}

El espesor de cromo duro depositado varió dependiendo del tiempo y de la densidad de corriente aplicada. El cálculo del espesor, en micras, se obtuvo a partir de la evaluación del volumen del depósito; aquel se determina con el peso de las probetas antes y después del proceso de cromado duro, restando el primer peso del segundo y dividiendo este resultado por la densidad del cromo junto al área superficial calculada, de la siguiente manera:

$$
\text { Espesor }=\left(\frac{\Delta M a s a}{\text { area } \cdot \rho C r}\right)
$$

A continuación se muestra en la Figura 4 el espesor alcanzado a partir de la evaluación del volumen de depósito para los grupos 1, 2, 3, 4 y 5 .

La combinación de parámetros combinados reflejó un mayor volumen depositado para el grupo 1, con espesor de $82.61[\mu \mathrm{m}]$. Esto se debe a que el grupo 1 fue sometido a la máxima densidad de corriente y al mayor tiempo empleado dentro de este experimento. Como se observa en la tabla 2, el grupo 1 está seguido del grupo 2, grupo 3 seguido del grupo 5, y finalmente el grupo 4. Es importante observar que el espesor del grupo 3 superó la mitad del espesor del espesor del grupo 2 en tan solo un tercio del tiempo empleado únicamente por una mayor densidad de corriente.

\begin{tabular}{cccc}
\hline Grupo & $\begin{array}{c}\text { Densidad } \\
\text { de } \\
\text { Corriente } \\
{\left[\text { Amp/dm }^{2}\right]}\end{array}$ & $\begin{array}{c}\text { Tiempo } \\
{[\mathbf{m i n}]}\end{array}$ & $\begin{array}{c}\text { Espesor } \\
{[\mu \mathbf{m}]}\end{array}$ \\
\hline 1 & 57 & 90 & 82.6 \\
2 & 39 & 90 & 56.8 \\
3 & 57 & 30 & 28.9 \\
4 & 39 & 30 & 15.9 \\
5 & 48 & 60 & 43.1 \\
\hline
\end{tabular}

Tabla 2: Espesor de Cr según los parámetros de caracterización, tiempo y densidad de corriente, para las probetas en grupos 1-5.

El incremento de la densidad de corriente aumenta el espesor del depósito de $\mathrm{Cr}$ en el mismo periodo de tiempo. Una mayor densidad de corriente logró depositar mayor espesor de cromo en menor tiempo [2]. Por lo tanto la velocidad del proceso de cromado aumenta al incrementar la densidad de corriente.

\section{Comportamiento del depósito según la ley de Fara- day}

Resulta evidente que la teoría de la electrodeposición se deriva a partir de la ley de Faraday. Dado que este es un proceso experimental, es posible determinar la eficiencia del proceso a partir de la ecuación de Faraday. Para ello, se determinó la carga suministrada de los grupos y se graficó de acuerdo a su espesor de cromo obtenido, así se obtuvo una regresión lineal tal como se muestra en la Figura 5.

A partir de la regresión lineal de la figura anterior se obtuvo la ecuación 7 que relaciona el espesor experimental obtenido con la densidad de carga. Cabe recalcar que el segundo término fue separado ya que si no existe densidad de carga no existe electro deposición.

$$
E_{\text {exp }}=2,60 \cdot 10^{6} \times Q
$$

Al resolver la ecuación de Faraday y reemplazar valores se obtuvo la siguiente ecuación:

$$
E_{\text {teorico }}=1,296 \cdot 10^{7} \times Q
$$

La división de estas dos ecuaciones, experimental para teórica determina la eficiencia del experimento bajo los parámetros establecidos de temperatura, ánodos, solución acida y material base. La eficiencia calculada fue del $20 \%$. Según el manual de ASM, la eficiencia del 


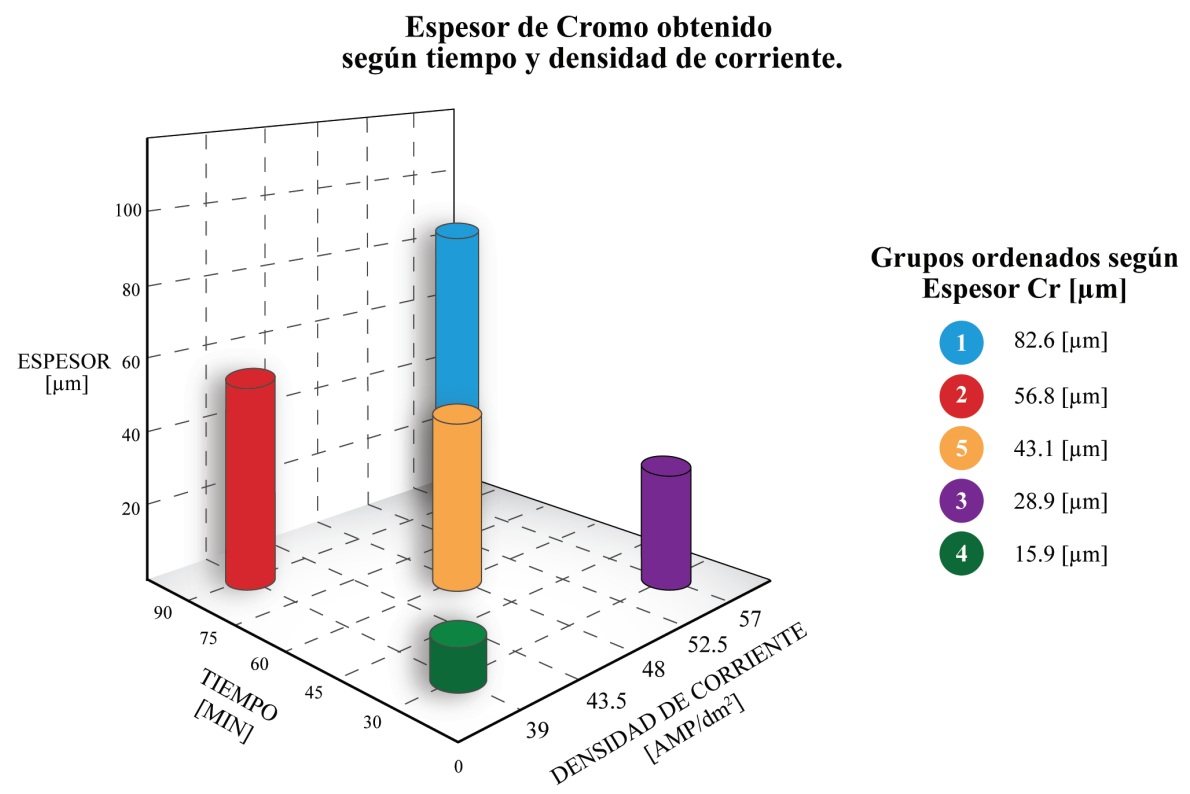

Figura 4: Espesor de Cr con distinta combinación de tiempo y densidad de corriente.

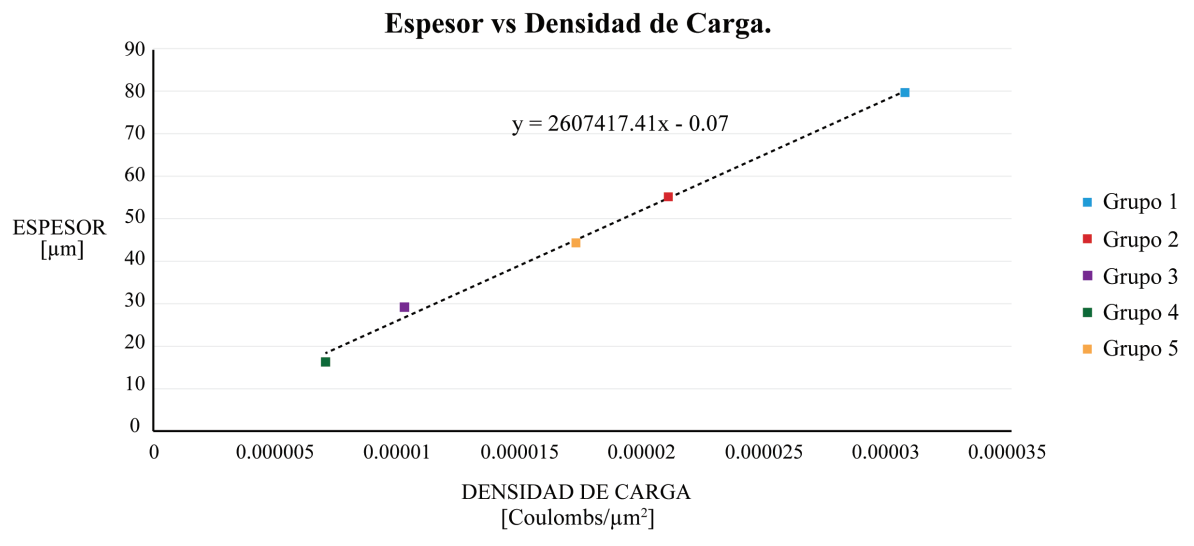

Figura 5: Espesor de $\mathrm{Cr}$ depositado vs densidad de carga / micrómetro cuadrado.

proceso de cromo duro varía dependiendo de la densidad de corriente y la temperatura de la solución. La eficiencia aumenta significativamente al aumentar la densidad de corriente [2]. La obtención del $20 \%$ de eficiencia se encuentra dentro del rango obtenible ya que el proceso de cromado involucra otras reacciones químicas dentro del proceso de electrolisis [11].

\section{Análisis Metalográfico}

Análisis de espesor, micro dureza y agrietamiento del cromo depositado fueron los parámetros examinados dentro del análisis metalográfico.

\section{Análisis de espesor}

La determinación del espesor de material depositado se comprobó al usar microscopía óptica con magnificación de 200x. El espesor de $\mathrm{Cr}$ fue de apariencia brillante en relación al material base de acero A36. Al final de la electro deposición se obtiene el depósito de cromo sobre el material base de acero A36 como se muestra en la Figura 6; la tabla 3 resume los resultados obtenidos y compara el espesor que fue obtenido mediante gravimetría.

\begin{tabular}{ccccc}
\hline Grupo & $\begin{array}{c}\text { Densidad } \\
\text { de } \\
\text { Corriente } \\
{\left[\mathbf{A m p} / \mathbf{d m}^{2}\right]}\end{array}$ & $\begin{array}{c}\text { Tiempo } \\
{[\mathbf{m i n}]}\end{array}$ & $\begin{array}{c}\text { Espesor } \\
\text { medido por } \\
\text { microscopía } \\
{[\mu \mathbf{m}]}\end{array}$ & $\begin{array}{c}\text { Espesor } \\
\text { medido por } \\
\text { gravimetría } \\
{[\mu \mathbf{m}]}\end{array}$ \\
\hline 1 & 57 & 90 & 81.9 & 82.61 \\
2 & 39 & 90 & 52.1 & 56.82 \\
3 & 57 & 30 & 27.0 & 28.89 \\
4 & 39 & 30 & 14.4 & 15.93 \\
5 & 48 & 60 & 41.3 & 43.12 \\
\hline
\end{tabular}

Tabla 3: Espesor de Cr medidos por microscopía y gravimetría para las probetas que representan los grupos 1-5.

La medición del espesor de cromo fue realizado en el centro de cada probeta. Las mediciones efectuadas fueron representadas en la tabla 3. Dichas, presentaron ciertas diferencias en relación a la tabla 2 (espesores obtenidos mediante gravimetría). La diferencia que se obtuvo fue mínima, sin embargo esto se debió a que existe una mayor cantidad de cromo depositado en las esquinas de 

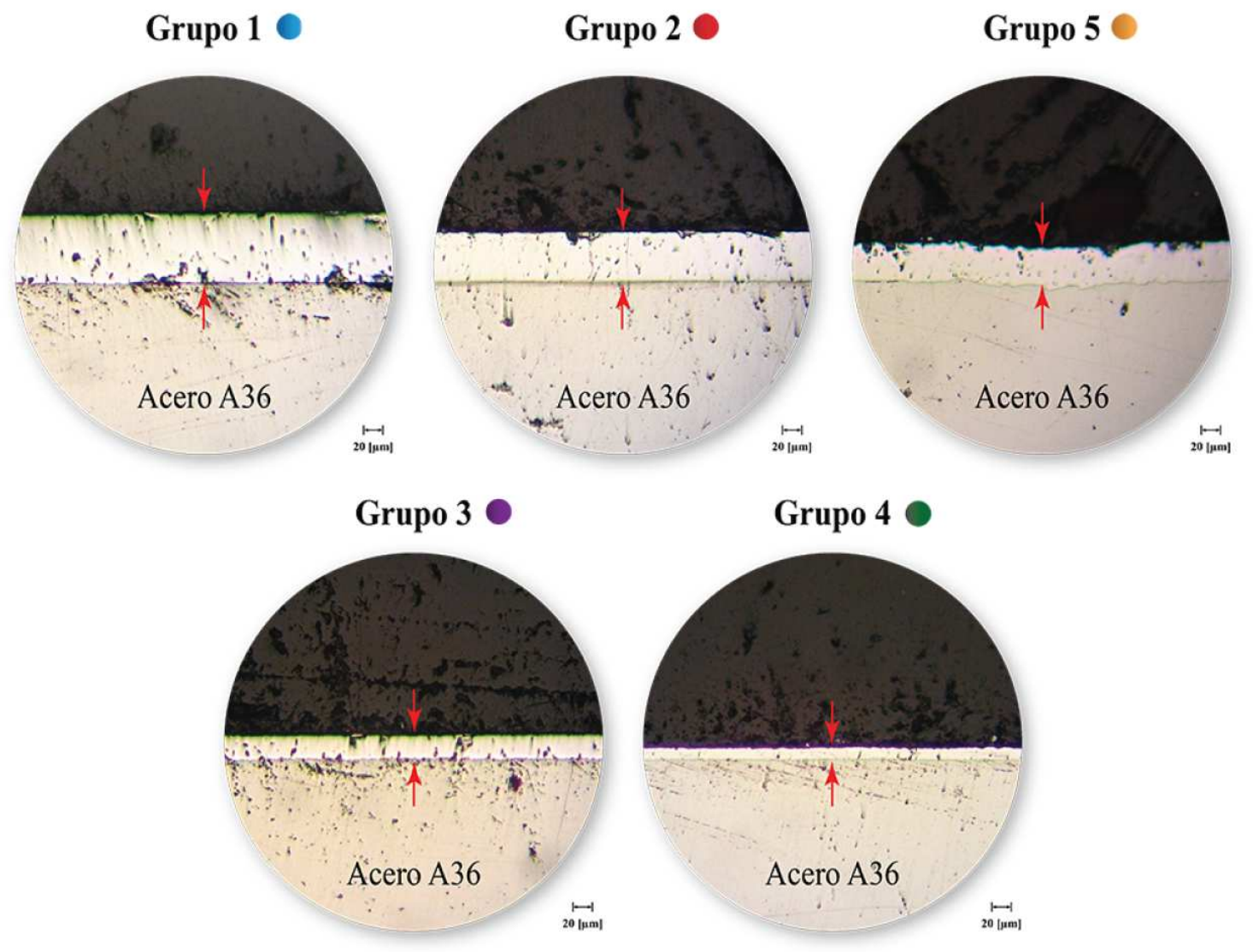

Figura 6: Sección Transversal del Cr depositado sobre material base acero AISI A36. Los grupos son alineados según la cantidad de cromo depositado para una mejor visualización. Las flechas rojas indican el espesor de cromo sobre acero A36.

la probeta ya que éstas actuaron como puntos concentradores de carga eléctrica durante el proceso de electrolisis. Se comprueba este hecho al haber examinado las esquinas de la probeta con el soporte del manual de la ASM que menciona que las esquinas y los picos existentes en el material base actúan como concentradores de carga eléctrica depositando mayor cantidad de material sobre dichos puntos [2].

\section{Análisis de micro-dureza}

El ensayo de micro-dureza se efectuó sobre cada grupo. La indentación se realizó sobre la superficie de cada probeta en la parte central de la misma. El resultado se obtuvo a partir de la medición de la longitud mayor de la indentación sobre la superficie del recubrimiento de cromo. Como muestra de lo mencionado, se observa en la Figura 7 la penetración de la punta Knoop sobre el recubrimiento de cromo.

La tabla 4 muestra los resultados que fueron obtenidos de la medición de dureza para cada uno de los grupos, los valores de dureza se representan en escala Knoop y Rockwell C.

El espesor depositado sobre los grupos dependió de la combinación de parámetros de densidad de corriente y tiempo aplicado. Los resultados de micro-dureza presentaron una alta dureza para el grupo 1; posteriormente

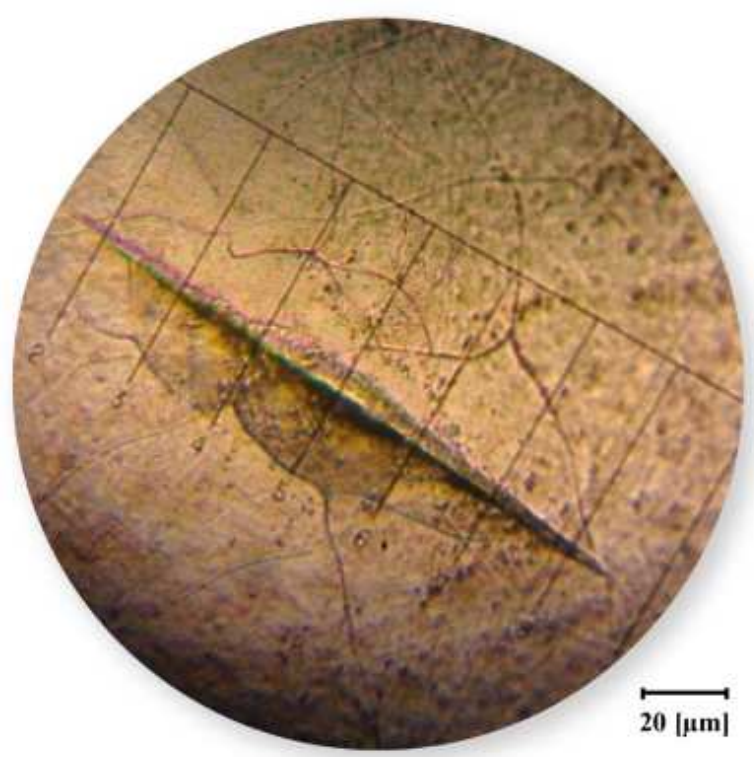

Figura 7: Indentación sobre la probeta representante al grupo 4 con una ampliación de 400x. Dureza Knoop 256 KHN.

para el grupo 2, grupo 3, grupo 5 y finalmente el grupo 4.

La micro dureza obtenida en el grupo 4 fue de 21 HRC, este valor es apartado del rango de micro-durezas del cromo (60 - 70 HRC). La baja densidad de corriente y 


\begin{tabular}{cccc}
\hline Grupo & $\begin{array}{c}\text { Longitud } \\
\text { indentación } \\
{[\mu \mathbf{m}]}\end{array}$ & $\begin{array}{c}\text { Knoop } \\
\text { Hardness }\end{array}$ & $\begin{array}{c}\text { Rockwell C } \\
\text { Hardness }\end{array}$ \\
\hline 1 & 85.2 & 981.0 & 70.2 \\
2 & 88.6 & 924.7 & 68.2 \\
3 & 92.4 & 833.3 & 66.0 \\
4 & 166.5 & 256.7 & 21.0 \\
5 & 90.5 & 868.0 & 65.9 \\
\hline
\end{tabular}

Tabla 4: Dureza Knoop y su equivalencia en escala Rockwell C presente en grupos 1-5.

el corto período de tiempo implicó la formación de una delgada capa de cromo sobre el material substrato, lo cual al momento de la indentacion, la punta sensibilizó el material substrato y contrastó una baja medición [8].

En complementación de este análisis y observar una posible tendencia de variación de dureza según la densidad de corriente aplicada y tiempo suministrado, se realizó mediciones de micro dureza en la sección transversal del recubrimiento de cromo, la tabla 5 muestra los resultados obtenidos.

\begin{tabular}{cccc}
\hline Grupo & $\begin{array}{c}\text { Longitud } \\
\text { indentación } \\
{[\mu \mathbf{m}]}\end{array}$ & $\begin{array}{c}\text { Dureza } \\
\text { Vickers }\end{array}$ & $\begin{array}{c}\text { Dureza } \\
\text { Rockwell C }\end{array}$ \\
\hline 1 & 22.4 & 1112.2 & 72.3 \\
2 & 24.5 & 926.6 & 67.7 \\
3 & 23.9 & 971.4 & 68.8 \\
5 & 24.8 & 904.8 & 67.1 \\
\hline
\end{tabular}

Tabla 5: Dureza transversal en Vickers y Rockwell C presente en grupos 1, 2, 3 y 5.

Se determinó mediante los resultados que el grupo 1 presenta mayor dureza, luego se encuentra el grupo 3, el grupo 2 y finalmente el grupo 5 . No se obtuvo un resultado para el grupo 4 debido a que el espesor de cromo depositado sobre la probeta es: 14.47 micrómetros, demasiado delgado para la punta del indentador Vickers. Los resultados anteriores muestran que la dureza disminuye a medida que la densidad de corriente cae. Es decir, para los grupos 1 y 3 se empleó una densidad de corriente equivalente a $57\left[\mathrm{~A} / \mathrm{dm}^{2}\right]$, mientras que el grupo $2,39\left[\mathrm{~A} / \mathrm{dm}^{2}\right]$ y para el grupo $5,48\left[\mathrm{~A} / \mathrm{dm}^{2}\right]$.

Las mediciones de micro-dureza que fueron realizadas en sentido superficial y transversal, presentaron variaciones de orden mínimo entre grupos. Se destaca que estadísticamente los valores individuales de cada medida son válidos. Las mediciones realizadas de forma transversal mostraron un ligero aumento respecto a las mediciones realizadas de forma superficial. La variación entre los valores correspondió a la presencia de grietas en la superficie del depósito orientadas en sentido de aplicación de la carga. Se comprueba posibles tendencias de densidad de grietas por área cuadrada según la variación de parámetros que influyeron sobre cada grupo. Este estudio es descrito en la siguiente sección "Agrietamiento del cromo depositado".

\section{Agrietamiento del cromo depositado}

El proceso de combinación de tiempo y densidad de corriente afectó el depósito del cromo sobre la superficie del acero A36. La formación de grietas sucedió durante el proceso de cromado al momento en el que la tensión interna fue mayor que la resistencia a la fractura del cromo. La tensión interna aumentó debido a la filtración de átomos de hidrogeno entre átomos de cromo; los átomos de hidrogeno tienden a alinearse con otros átomos de hidrogeno y al momento en el que se difunden crean tensiones internas entre los átomos de cromo, hasta que la sumatoria de varias tensiones superan la resistencia a la tracción de cromo y este cede produciendo grietas aliviando tensiones [11].

Se realizó el siguiente análisis donde la superficie de las probetas es ampliada a 230x y 400x para observar la cantidad de grietas presentes según los parámetros empleados sobre cada grupo. Dichos conjuntos de moléculas de cromo depositados en forma cristalina delimitados por grietas son denominados clústeres. Se contabilizó el número de clústeres presentes dentro de un círculo de 200 micrones de diámetro. Fueron señalados, con color verde, los clústeres que se encuentran dentro del círculo y, con color morado los que se encuentran en la frontera del círculo. La Figura 8 presenta dichos clústeres para cada grupo mientras que el resultado de esta contabilización fue almacenado en la tabla 6 .

\begin{tabular}{cccc}
\hline Grupo & $\begin{array}{c}\text { Número de } \\
\text { clústeres } \\
\text { dentro del } \\
\text { círculo }\end{array}$ & $\begin{array}{c}\text { Número de } \\
\text { clústeres } \\
\text { en frontera } \\
\text { del círculo }\end{array}$ & $\begin{array}{c}\text { Cantidad de } \\
\text { clúster por } \\
{\left[\mathbf{m m}^{2}\right]}\end{array}$ \\
\hline 1 & 6 & 14 & 413.8 \\
2 & 10 & 14 & 541.1 \\
3 & 16 & 18 & 795.8 \\
4 & 27 & 20 & 1177.8 \\
5 & 18 & 17 & 843.5 \\
\hline
\end{tabular}

Tabla 6: Cantidad de clústeres medidos ópticamente para las probetas que representan los grupos 1-5.

La menor cantidad de grietas se encuentra presente en el grupo 1, luego el grupo 2, grupo 3, grupo 5 y finalmente el grupo 4. El orden de dureza que ha sido obtenida para cada grupo según la tabla 4 , es inversamente proporcional a la cantidad de grietas existentes en la probeta. Las mediciones realizadas de dureza sobre las probetas de manera superficial (indentación perpendicular a la superficie), refleja una menor cantidad de grietas sobre la superficie y una alta densidad de corriente empleada [2]. Una tendencia similar se observa para las mediciones de micro dureza que fueron realizadas transversalmente, sin embargo la densidad de corriente y el tiempo son las únicas variables de incidencia ya que las grietas únicamente alteran la medición para la medición superficial. Según los resultados que fueron obtenidos de este estudio, la cantidad de grietas disminuye según aumenta la densidad de corriente en combinación con el tiempo, la Figura 9 grafica claramente este hecho. 

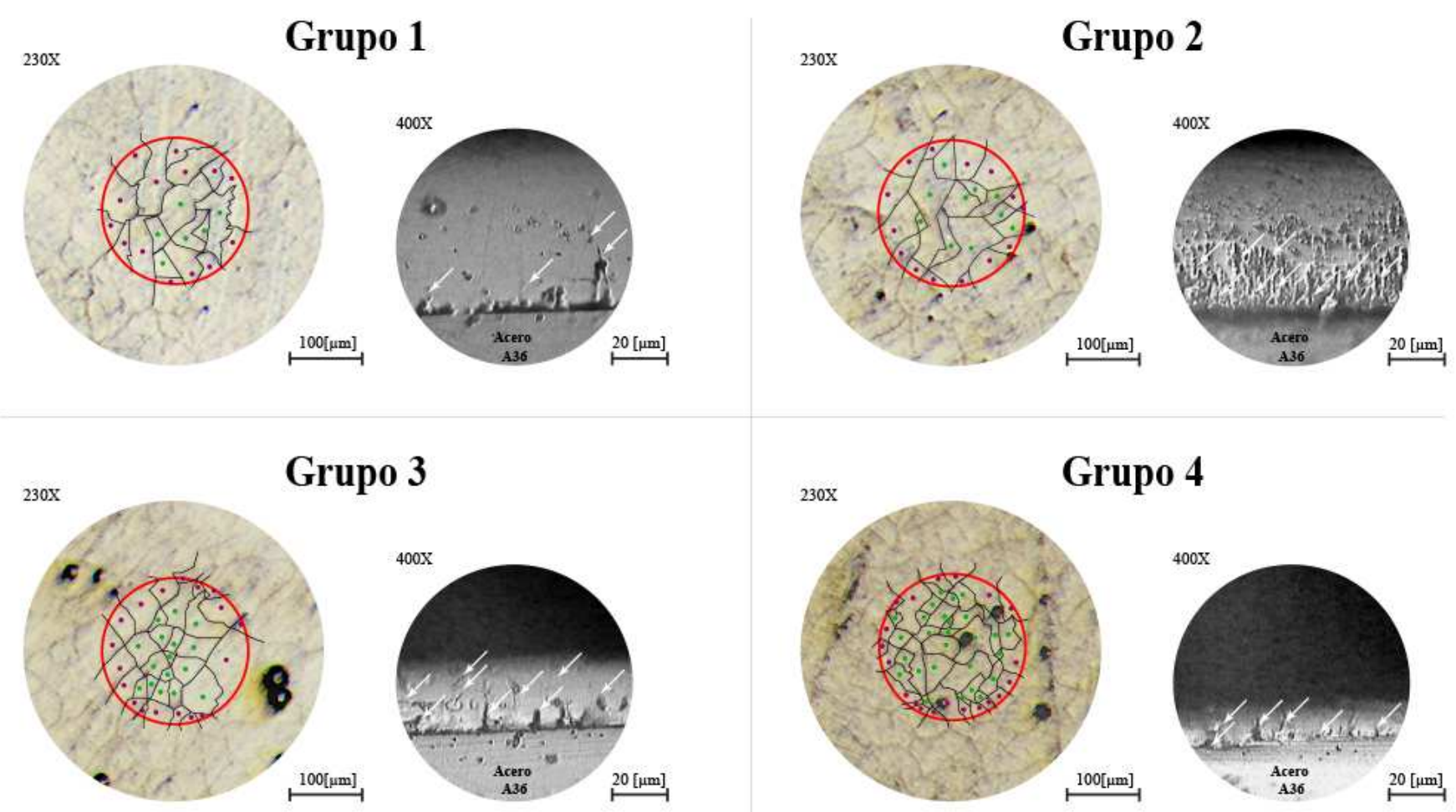

\section{Grupo 3}

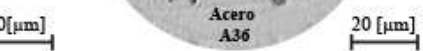
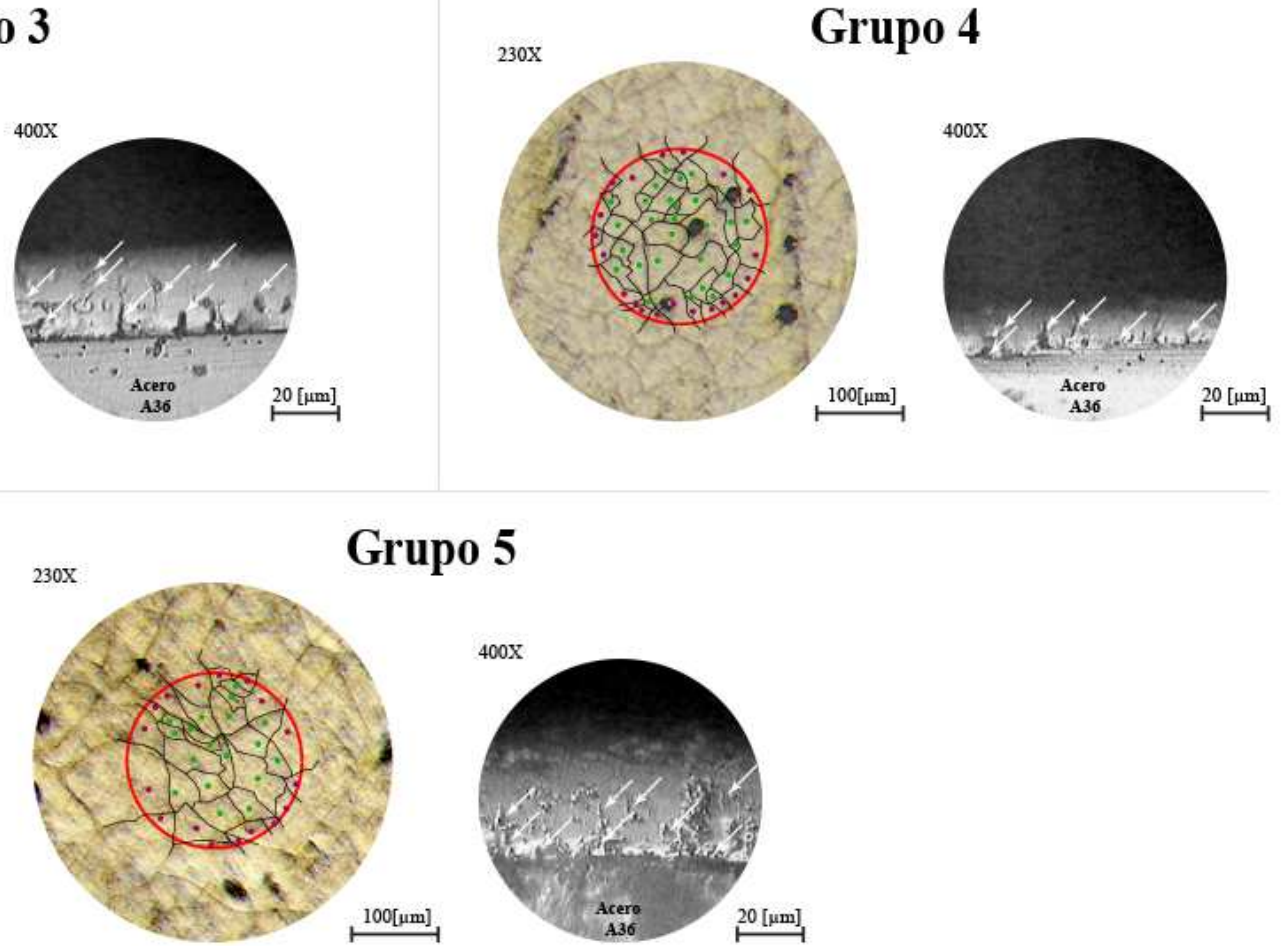

Figura 8: Grietas laterales y clústeres de Cr enmarcados para las probetas pertenecientes a los grupos 1-5.

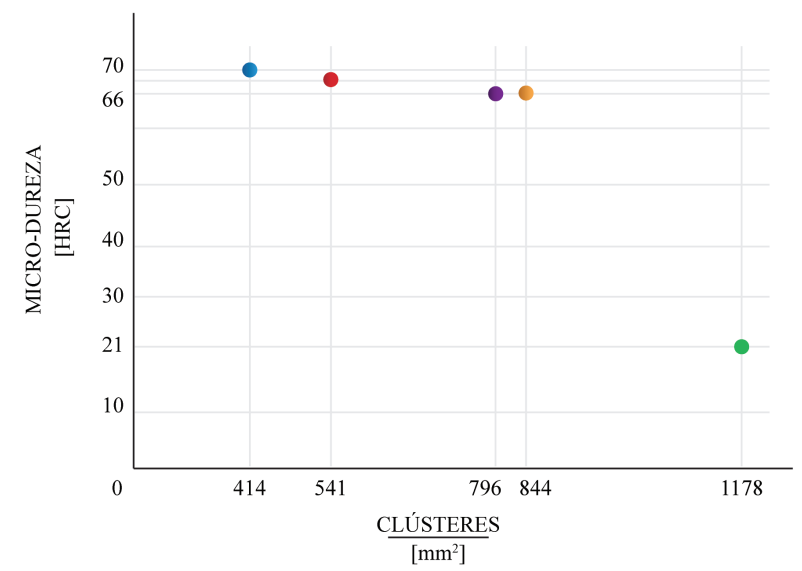

(a)

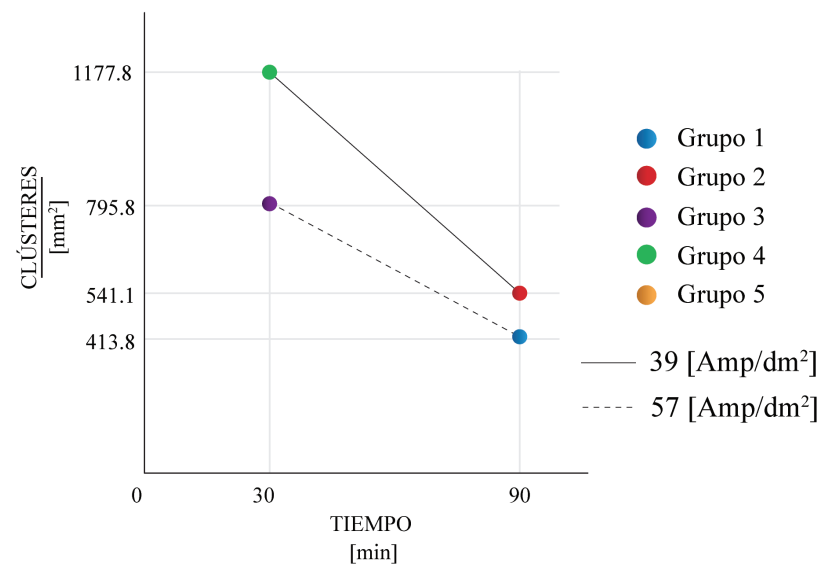

(b)

Figura 9: (a) Micro-dureza superficial (indentación perpendicular a la superficie) vs Cantidad de Clústeres superficiales. (b) Cantidad de Clústeres superficiales vs tiempo suministrado a diferentes densidades de corriente. 
Al emplear una menor densidad de corriente, se presentó una mayor cantidad de grietas y, como consecuencia una menor micro-dureza superficial. Sin embargo, la aplicación de una mayor cantidad de tiempo, afectó en la reducción del número de grietas superficiales, ya que al emplear una misma densidad de corriente durante un período de tiempo prolongado, la cantidad de grietas se vio reducida. Debido a ello se toma a consideración que la exposición prolongada de tiempo dentro del baño electrolítico depositó una fase de cromo más uniforme que la anterior, con ello las grietas anteriores son cubiertas y el número de las mismas fueron reducidas en cuanto se aproxima a la superficie del depósito de cromo.

En la Figura 8, se muestra con una magnificación de 400X, las secciones transversales de las probetas, clasificadas según sus agrupaciones. Se observa que el grupo 1 y el grupo 3 fueron conectados con la misma densidad de corriente, pero a medida que la cantidad de depósito de $\mathrm{Cr}$ aumenta, el número de grietas disminuye.

La Figura 9(b) muestra la comparación entre el tiempo suministrado y la reducción de clústeres superficiales según se incrementa el tiempo para las densidades de corriente equivalentes a: 39 y $57\left[\mathrm{Amp} / \mathrm{dm}^{2}\right]$. El tiempo, representó un cambio significativo en cuanto a la obtención de clústeres superficiales en ambas comparaciones. En ambos casos, la cantidad de clústeres obtenida es reducida casi por la mitad al triplicar el período de tiempo de electro-deposición. Es importante mencionar que a una menor cantidad de grietas la micro-dureza superficial aumenta. Por tanto, la relación que existe entre tiempo y micro-dureza superficial se debe a la cantidad de clústeres formados y existe menor cantidad de clústeres mientras mayor sea la densidad de corriente empleada. Entonces, se verificó con la Figura 9(a) que la dureza superficial del depósito de cromo aumentó a medida que la densidad de corriente y el tiempo se incrementaron. La dureza transversal no es considerada debido a que la orientación de las grietas y la magnitud de la carga no presentaron necesariamente una relación entre el campo de deformación y las grietas.

\section{Conclusiones}

En el presente artículo se describe los pasos preliminares del proceso de cromo duro para 5 condiciones de combinación de parámetros de densidad de corriente y tiempo. Se obtuvo 4 mediciones de micro-dureza dentro del rango 65 - 70 Hard Rockwell C previsto; estos fueron los grupos 1, 2, 3 y 5. La medición de microdureza del grupo 4 es de 21 HRC, medición apartada del rango de micro-durezas. Los factores que influyeron en la obtención de este resultado fueron la baja densidad de corriente empleada y el corto período de tiempo suministrado concibiendo como resultado la formación de una delgada capa de cromo depositada sobre el material substrato. Se logró además encontrar la eficiencia experimental y la ecuación empírica de depósito de masa según la carga aplicada a partir de la ley de electrodeposición de Faraday para los parámetros de trabajo local.

La eficiencia encontrada muestra la versatilidad que ofrece este procedimiento al combinar el tiempo y densidad de corriente. Sin embargo, los resultados obtenidos mostraron un mejor desempeño al momento de utilizar altas densidades de corriente durante períodos prolongados de tiempo. Al utilizar alta densidad de corriente y mayor tiempo, la cantidad de grietas superficiales fue reducida, al reducir la cantidad de grietas se presentó un incremento de dureza superficial del recubrimiento de cromo. Las mediciones de micro-dureza transversal presentaron una tendencia similar al emplear una elevada densidad de corriente, a medida que se incrementó la densidad de corriente la dureza transversal paralela a la superficie incrementó.

\section{Agradecimientos}

Los autores agradecen a Cesar Zambrano, director del colegio de ciencias e ingeniería de la Universidad san Francisco de Quito por su apoyo desinteresado durante esta investigación. Se agradece a los miembros del laboratorio de la Escuela Politécnica del Ejército por su soporte en implementos de laboratorio. El presente estudio tiene la base y el soporte en la Universidad San francisco de Quito a través de sus fondos de financiamiento del Chancellor Grant-USFQ 2013, utilizado para esta investigación.

\section{Referencias}

[1] Kostylev, A.; Pokrovsky, Y.; Lumpov, A. 2012. "Advanced Chromium Carbide Coatings on Piston Rings by CVD: A Highly Adaptable new method with relatively low cost". Cengage Learning: 2-26.

[2] American Society for Metals. 1994. "Handbook of Surface Engineering". ASM International.

[3] Birnbaum, H. K. 1986. "Hydrogen Embrittlement”. Pergamon Press.

[4] U.S. Environmental Protection Agency. 1998. "Toxicological Review of Hexavalent Chromium”. Washington, DC., United States: National Center for Environmental Assessment, Office of Research and Development.

[5] Jewett, R. P.; Walter, R. J.; Chandler, W. T.; Frohmberg, R. P. 1973. "Hydrogen environment embrittlement of metals". Canoga Park, CA: NASA United States.

[6] Ellor, J.; Young, W. 2004. "Thermally Sprayed Metal Coatings to Protect Steel Pilings: Final Report and Guide". Washington DC: Transportation Research Board (528).

[7] Industria Metalquímica Galvano. 2011. "Boletín técnico Sistema de Cromo Di-Co”. Quito: 1-2. 
[8] American Society for Testing and Materials. 2005. "Guide for Preparation of Metallographic Specimens" ASTM E3-01: 1-12.

[9] American Society for Testing and Materials. 2005. "Practice for Microetching Metals and Alloys". ASTM E407: 1-21.

[10] Such, T. E.; Dennis, J. K. 1993. "Nickel and Chromium Plating", Third Edition ed. Ohio, United States: Woodhead Publishing Ltd \& ASM International.

[11] American Society for Testing and Materials. 2005. "Standard Test Method for Microindentation Hardness of Materials". ASTM E384: 1-24. 